\section{Colourful grounds for separation}

\author{
George H. Elder
}

Biomedical Chromatography. Editors-inchief Elizabeth F. Hounsell and C.K. Lim. Heyden, Spectrum House, Hillview Gardens, London NW4 2JQ, UK. 6/yr. UK 1120 ; elsewhere $\$ 180$.

THE title of this new journal reflects its contents. Each issue contains up to a dozen concise papers describing chromatographic methods for the separation of substances of biological interest and commenting on their applications; reviews are also published occasionally.

Many of the papers have a clinical slant and there is a strong bias towards highperformance liquid chromatography. But the range of analytes discussed is commendably wide; the editors have included papers on proteins, peptides, amino acids, carbohydrates, steroids, porphyrins and vitamins, while managing to control the advancing tide of drug separations sufficiently to preserve balance.

The production quality is high, the turnround time of six months or under is good, and the editors appear to have achieved their aims of attracting contributions from many countries and encouraging proper description of methods. I could find none of the all-too-frequent accounts of HPLC separations in which the description of solvents is so vague as to almost leave to the reader the task of devising the method.

Chromatographic separations are central to much biomedical research and to many diagnostic and monitoring methods. Where does one turn when faced with a separation problem? Most of us hope that it will have already been at least partially solved and consult colleagues and the literature. The latter contains journals devoted exclusively to chromatography, analytical journals with a wider scope and the methods sections of papers in general journals.

Apart from extending the opportunities for publication, does Biomedical Chromatography have anything to offer? Most of the papers, as is perhaps to be expected from the way that method-orientated research now operates, are in the "state of the art" rather than the "never separated before" category. There is a need for a journal emphasizing medical applications of chromatography; but the competition is fierce from established, though less specific, publications. High standards and prestige will be required: Biomedical Chromatography shows promise.

George H. Elder is Professor in the Department of Medical Biochemistry, University of Wales College of Medicine, Heath Park, Cardiff CF4 $4 X N, U K$

\title{
Image promotion
}

George K. Radda

American Journal of Physiologic Imaging. Editor-in-chief Maynard L. Freeman. Alan R. Liss. 4/yr. USA \$95; elsewhere $\$ 108$.

THis new journal is aimed at "an audience interested in the study of physiology and pathophysiology by imaging techniques and their effects on the practice of medicine". The editorial in the first issue also states that the journal intends to cut across traditional disciplines.

Both of these aims are highly laudable, and if achieved will certainly result in a scientific periodical that differs from the highly specialized journals publishing papers on different aspects of medical imaging. The emphasis on physiological investigations is also welcome. The brief editorial statement in the fourth issue, reporting a discussion by the editorial board as to whether the term "physiologic" was too restrictive and whether

\section{Fertile imagination}

\section{R. Austin}

Human Reproduction. Executive editor R. G. Edwards. IRL. 8/yr. $\$ 160$ (institutional); $\$ 80$ (individual).

Human procreation has become a fascinatingly complex research area, ranging through a broad spectrum of medical and biological interests. Problems being studied include sexual differentiation and development, reproductive and social behaviour, fertility limitation and promotion, gamete and embryo manipulation and storage, pregnancy induction and maintenance, fetal physiology, birth processes and diagnosis, and the prevention and treatment of birth defects. And most of these topics also have their comparative, legal and sociological aspects.

Human Reproduction is designed to provide a forum for the exchange of information and ideas on all these matters. Acceptable material takes the form of reports of original basic and clinical research, reviews of books and other technical publications, verbatim reports of select assemblies (for example those of WHO on "Artificial Reproduction", and of the Council of Europe on "New Techniques in Human Fertilization and Embryology" and "The Use of Human Embryos and Fetuses"), and commentaries and short communications. In addition, this journal is the official organ of the European Society of Human Reproduction and Embryology, and biochemical, metabolic etc. should be added to the title, indicates the concern of the board to include papers on functional investigations in the broadest sense.

The papers published are original articles and "Current Concepts - Review Articles" (two reviews per issue). The journal is well printed, and with the highquality production had a good start. Some papers have been accepted rapidly (within a month of submission), and overall publication times are very commendable.

To date, however, the spread of the papers has not been as broad as the stated aims. Most contributions are in the general field of nuclear medicine with some limited contributions in nuclear magnetic resonance, computed tomography and echocardiography. It would be sad if the editorial board cannot in future attract papers outside the use of isotopes. On the whole, the journal in its present format is good value even if I intensely dislike the term "physiologic imaging".

George K. Radda is British Heart Foundation Professor of Molecular Cardiology, Department of Biochemistry, University of Oxford, South Parks Road, Oxford OXI $3 Q U, U K$.

special supplements present the abstracts from the annual conferences of the society, and occasionally the conferences of other relevant groups. Publication is only in English, but with the commendable editorial policy that poor English is not in itself grounds for rejecting a paper, and that every effort is made to render the effusion comprehensible.

Human Reproduction was born in January 1986. It is edited by R.G. Edwards, the prime mover in successful human in vitro fertilization and embryo transfer, aided by associate editors and an editorial board of Western Europeans known for

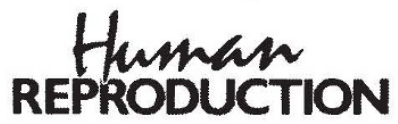

their contributions in the journal's area of interest. It is a good-looking product, roughly A4 in page size, with two columns of clear print that makes for comfortable reading, and high quality half-tones and line drawings. Each paper begins with a helpful summary in bold type.

Articles I selected for close inspection all seemed well worthy of their inclusion. I was also favourably impressed with the speed of publication - the 131 refereed communications in the first 11 issues appeared in print on average 3.7 months after receipt. In brief, my opinion is that the circulation of Human Reproduction deserves to grow in a manner consistent with the journal's title.

C.R. Austin, 47 Dixon Road, Buderim, Queensland 4556, Australia, is Emeritus Charles Darwin Professor of Animal Embryology in the University of Cambridge. 